\title{
Evaluación de la accesibilidad de páginas web de universidades españolas y extranjeras incluidas en rankings universitarios internacionales
}

\author{
José R. Hilera*, Luis Fernández*, Esther Suárez*, Elena T. Vilar** \\ *Universidad de Alcalá. Madrid, España. Correo-e: jose.hilera@uah.es; luis.fernandezs@uah.es; esther_su6@hotmail.com. \\ **SERMAS. Alcalá de Henares. Madrid, España. Correo-e: evilar.gapm03@salud.madrid.org.
}

Recibido: 18-11-2011; 2a versión: 18-05-2012; Aceptado: 19-06-2012.

Cómo citar este artículo/ Citation: Hilera, J.R.; Fernández, L.; Suárez, E.; Vilar, E.T. (2013). Evaluación de la accesibilidad de páginas web de universidades españolas y extranjeras incluidas en rankings universitarios internacionales. Revista Española de Documentación Científica, 36(1):e004. doi:http://dx.doi.org/10.3989/redc.2013.1.913

Resumen: En este artículo se describe un estudio llevado a cabo para evaluar la accesibilidad de los contenidos de los portales Web de algunas de las universidades extranjeras y españolas más relevantes según tres rankings universitarios publicados. Se han seleccionado universidades incluidas simultáneamente en "Academic Ranking of World Universities", en "Times Higher Education Supplement" y en "Webometrics Rankings of World Universities". La evaluación de la accesibilidad se ha realizado comprobando el cumplimiento de las pautas de accesibilidad de contenidos Web establecidas por el World Wide Web Consortium en la recomendación WCAG 2.0. Se ha tratado de determinar si universidades con prestigio a nivel académico ofrecen la información sobre la universidad a través de su Web, de tal forma que pueda ser visitada por todas las personas (profesores, estudiantes), independientemente de si tienen o no algún tipo de discapacidad. Desafortunadamente los resultados no han sido tan buenos como cabría esperar de universidades de tanta importancia.

Palabras clave: Accesibilidad de contenidos Web; usabilidad; ranking universitario; discapacidad; WCAG 2.0.

\section{Accessibility assessment of web pages of Spanish and foreign universities included in international rankings}

Abstract: This article describes a study conducted to evaluate the accessibility of the contents of the Web sites of some of the most important universities in Spain and the rest or the world, according with three international rankings. Universities included simultaneously in "Academic Ranking of World Universities", in "Times Higher Education Supplement" and in "Webometrics Rankings of World Universities" have been selected. The accessibility assessment has been carried out to check compliance with accessibility guidelines for Web content established by the World Wide Web Consortium recommendation in WCAG 2.0. We have tried to determine whether universities with greater academic prestige have been concerned to provide accesible information about the university through its website so that it can be accessed by everyone (teachers, students), regardless of whether or not the user has a disability. Unfortunately the results have not been as good as expected from universities as important.

Keywords: Web content accessibility; usability; ranking of universities; disability; WCAG 2.0.

Copyright: () 2013 CSIC. Este es un artículo de acceso abierto distribuido bajo los términos de la licencia Creative Commons Attribution-Non Commercial (by-nc) Spain 3.0. 


\section{INTRODUCCIÓN}

La accesibilidad indica la facilidad con la que algo puede ser usado, visitado o accedido en general por todas las personas, especialmente por aquellas que poseen algún tipo de discapacidad (BOE, 2003). La accesibilidad web se refiere a la facilidad de que todas las personas puedan percibir, entender, navegar e interactuar con la web. Existen multitud de normas y estándares en el diseño de páginas web encaminadas a facilitar la consecución de un adecuado nivel de accesibilidad (Serrano y otros, 2010).

Entre los esfuerzos de normalización, destaca la Web Accesibility Initiative del World Wide Web Consortium (W3C), que trata de establecer recomendaciones para conseguir contenidos, navegadores, entornos de desarrollo de web accesibles. Entre sus recomendaciones se encuentran las Web Content Accesibility Guidelines (WCAG), o conjunto de pautas para conseguir páginas web accesibles. La última versión de esta recomendación es WCAG 2.0 (W3C, 2008), adoptada en 2012 por ISO como estándar (ISO, 2012), y el estudio llevado a cabo en este artículo se ha basado precisamente en esta última versión, que establece un total de 12 pautas a seguir (tabla I). Estas doce pautas no son testeables en sí, sino que proporcionan los objetivos básicos que los autores deben lograr con objeto de crear un contenido más accesible para los usuarios con discapacidades. Para cada pauta se proporcionan los criterios de éxito verificables que permiten emplear las pautas en aquellas situaciones en las que existan requisitos y necesidad de comprobación de conformidad.

Para cada una de las pautas y criterios de éxito, el W3C ha documentado también una amplia va- riedad de técnicas. Cada pauta tiene asignada una prioridad que indica cómo afecta a la accesibilidad de un sitio web si dicho punto de verificación no se cumple. Las prioridades son las siguientes:

- Prioridad 1: Son aquellos criterios de éxito que un desarrollador web tiene que cumplir ya que, de otra manera, ciertos grupos de usuarios no podrían acceder a la información del sitio web.

- Prioridad 2: Son aquellos criterios de éxito que un desarrollador web debería cumplir ya que, si no fuese así, sería muy difícil acceder a la información para ciertos grupos de usuarios.

- Prioridad 3: Son aquellos criterios de éxito que un desarrollador web debería cumplir ya que, de otra forma, algunos usuarios experimentarían ciertas dificultades para acceder a la información.

Dependiendo del grado de cumplimiento de los criterios de éxito por un determinado sitio web existen tres niveles de conformidad (W3C, 2008):

- "A": Se satisfacen todos los criterios de éxito de prioridad 1.

- "AA": Se satisfacen todos los criterios de éxito de prioridad 1 y 2.

- "AAA": Se satisfacen todos los criterios de éxito de prioridad 1,2 y 3.

Aunque WCAG es sólo una recomendación, en países como España o Estados Unidos se ha convertido es un estándar de obligado cumplimiento. Así, en España, la legislación exige el cumplimiento del nivel de conformidad "AA" de WCAG 1.0 (W3C, 1999) para las páginas web de: la Administración Pública, entidades y empresas que se encarguen

Tabla I. Pautas de accesibilidad de contenido web establecidas en WCAG 2.0 (traducción disponible en http://www.sidar.org/traducciones/wcag20/es/)

\begin{tabular}{|c|c|}
\hline Número pauta & Texto original pauta \\
\hline 1.1 & $\begin{array}{l}\text { Proporcionar alternativas textuales para todo contenido no textual de modo que se pueda convertir a } \\
\text { otros formatos que las personas necesiten, tales como textos ampliados, braille, voz, símbolos o en un } \\
\text { lenguaje más simple. }\end{array}$ \\
\hline 1.2 & Proporcionar alternativas para los medios tempodependientes. \\
\hline 1.3 & $\begin{array}{l}\text { Crear contenido que pueda presentarse de diferentes formas (por ejemplo, con una disposición más } \\
\text { simple) sin perder información o estructura. }\end{array}$ \\
\hline 1.4 & Facilitar a los usuarios ver y oír el contenido, incluyendo la separación entre el primer plano y el fondo. \\
\hline 2.1 & Proporcionar acceso a toda la funcionalidad mediante el teclado. \\
\hline 2.2 & Proporcionar a los usuarios el tiempo suficiente para leer y usar el contenido. \\
\hline 2.3 & No diseñar contenido de un modo que podría provocar ataques, espasmos o convulsiones. \\
\hline 2.4 & $\begin{array}{l}\text { Proporcionar medios para ayudar a los usuarios a navegar, encontrar contenido y determinar dónde se } \\
\text { encuentran. }\end{array}$ \\
\hline 3.1 & Hacer que los contenidos textuales resulten legibles y comprensibles. \\
\hline 3.2 & Hacer que las páginas web aparezcan y operen de manera predecible. \\
\hline 3.3 & Ayudar a los usuarios a evitar y corregir los errores. \\
\hline 4.1 & $\begin{array}{l}\text { Maximizar la compatibilidad con las aplicaciones de usuario actuales y futuras, incluyendo las ayudas } \\
\text { técnicas. }\end{array}$ \\
\hline
\end{tabular}


de gestionar servicios públicos, de empresas privadas que reciban financiación pública, y de cualquier otra empresa con más de 100 trabajadores o una facturación superior a 6 millones de euros (BOE, 2007a). Por tanto, las universidades tienen obligación del cumplirlas.

En este artículo se analiza un grupo representativo de páginas web de los portales de las siete universidades españolas incluidas simultáneamente en tres conocidos rankings mundiales de instituciones universitarias, con objeto de comprobar el grado de cumplimiento de las recomendaciones WCAG 2.0. También se hace lo propio con los sitios web de algunas de las universidades más importantes a nivel mundial. Aunque la legislación en los países a los que pertenecen las universidades exige el cumplimiento de WCAG 1.0 , se ha realizado el estudio de la nueva versión 2.0 , por entender que en breve la legislación se actualizará para exigir el cumplimiento de la versión más reciente.

\section{TRABAJOS RELACIONADOS}

Se han publicado numerosos estudios de la accesibilidad de sitios web en diferentes áreas temáticas y países. Algunos de los más recientes se muestran en la tabla II.
Estos estudios se caracterizan por la aplicación de diferentes metodologías para la evaluación de la accesibilidad. El W3C está elaborando una metodología estándar de evaluación del cumplimiento de las pautas WCAG 2.0 por los sitios web, que próximamente será publicada como la recomendación WCAG-EM (W3C, 2012). Autores como Brajnik (2009) han publicado estudios comparativos de diferentes métodos de evaluación de la accesibilidad web, que suelen clasificarse en las siguientes categorías: métodos de evaluación de la accesibilidad: Conformance Review; Subjective Assessment, Screening, Barrier Walkthrough o User testing (Brajnik, 2008). En este trabajo se ha aplicado el método definido por el Instituto Nacional de Tecnologías de las Comunicaciones (INTECO, 2008) y probado en casos reales. Como ocurre en la mayoría de estudios publicados (tabla II), este método sería de la categoría Conformance Review, ya que un experto es quien realiza la evaluación, empleando un criterio para la elección de las páginas web a analizar, utilizando las herramientas pertinentes para la validación, que incluyen también listas de checkpoints o criterios de éxito, y realizando una comparación de los resultados (Serrano y otros, 2010).

Tabla II. Trabajos recientes sobre casos de evaluación de accesibilidad de sitios web

\begin{tabular}{|c|c|}
\hline Caso de evaluación & Referencia \\
\hline accessibility audit of Australian University websites & (Alexander y Rippon, 2007) \\
\hline $\begin{array}{l}\text { Evaluación comparativa de la accesibilidad de los espacios web de las bibliotecas } \\
\text { universitarias españolas y norteamericanas }\end{array}$ & $\begin{array}{l}\text { (Caballero-Cortés y otros, } \\
\text { 2009) }\end{array}$ \\
\hline Accesibilidad de las Páginas de Internet de las principales empresas españolas & (CERMI, 2010) \\
\hline Assessment of the Status of eAccessibility in Europe & (Comisión Europea, 2008) \\
\hline $\begin{array}{l}\text { Accessibility of European Commission Websites - Analysis of current status and } \\
\text { recommendations for improvement }\end{array}$ & (Comisión Europea, 2009a) \\
\hline $\begin{array}{l}\text { Web accessibility in European countries: level of compliance with latest international } \\
\text { accessibility specifications, notably WCAG 2.0, and approaches or plans to implement those } \\
\text { specifications }\end{array}$ & (Comisión Europea, 2009b) \\
\hline $\begin{array}{l}\text { Estudio comparativo de accesibilidad web en portales informativos de universidades } \\
\text { peruanas de educación a distancia }\end{array}$ & (Córdova, 2012) \\
\hline Accesibilidad web en los portales de los Ministerios de España & (Discapnet, 2009a) \\
\hline Accesibilidad en los portales web de Servicios y Plataformas de eLearning & (Discapnet, 2009b) \\
\hline Accesibilidad de Portales web Universitarios de España & (Discapnet, 2010) \\
\hline Accesibilidad de Plataformas de Redes Sociales & (Discapnet, 2011) \\
\hline $\begin{array}{l}\text { Evaluación de la accesibilidad de portales web en instituciones educativas en el área de } \\
\text { Centroamérica }\end{array}$ & $\begin{array}{l}\text { (Hernández y Amado- } \\
\text { Salvatierra, 2012) }\end{array}$ \\
\hline $\begin{array}{l}\text { Access to Information for Students with Disabilities; An Evaluation of the } 2010 \text { U.S. News and } \\
\text { World Report's Ranking of the Nation's Best Colleges and Universities }\end{array}$ & (Hill, 2010) \\
\hline Estudio Diagnóstico de Accesibilidad y Calidad web de la PYME Española & (INTECO, 2008) \\
\hline Informe sobre la accesibilidad de los sistemas de mensajería instantánea & (INTECO, 2009) \\
\hline A Web Accessibility Report Card for Top International University Web Sites & (Kane y otros, 2007) \\
\hline Accessibility and readability of university websites in Finland & (Karhu y otros, 2012) \\
\hline Accesibilidad de plataformas web comerciales en España & (Serrano y otros, 2010) \\
\hline La accesibilidad de las webs de las universidades españolas. Balance 2001-2006 & (Ribera y otros, 2009) \\
\hline $\begin{array}{l}\text { Web accessibility: A longitudinal study of college and university home pages in the } \\
\text { northwestern United States }\end{array}$ & \\
\hline
\end{tabular}


La mayoría de los estudios evalúan la accesibilidad de contenidos web según el estándar WCAG 1.0; sin embargo, no es el único estándar que puede aplicarse en este contexto. En la tabla III se recogen algunos otros estándares que pueden ser de aplicación. En la evaluación realizada en este artículo, se ha decidido aplicar WCAG 2.0, aunque la legislación española y de otros países todavía no exige su cumplimiento, pero será la referencia en el futuro en este contexto. En el caso español, en 2012 se ha publicado la nueva versión de la norma UNE 139803 (AENOR, 2012), que es una traducción de WCAG $2.0 \mathrm{y}$, en consecuencia, toda la legislación ligada actualmente a la versión de 2004 de la norma UNE, lo estará en el futuro próximo a la nueva versión, y por tanto a WCAG 2.0.

La principal novedad del trabajo que aquí se presenta frente a otros análisis de universidades españolas (Discapnet, 2010; Ribera y otros, 2009) o extranjeras (Alexander y Rippon, 2007; Hill, 2010; Kane y otros, 2007; Thompson y otros, 2010), además de aplicar la nueva versión WCAG 2.0, es que incluye la evaluación simultánea de universidades españolas y extranjeras; lo cual permite la comparación de los resultados en ambo ámbitos. En algunos de los estudios citados se ha realizado la selección de universidades a partir de un ranking (Hill, 2010; Kane y otros, 2007). En nuestro trabajo se han utilizado tres rankings en la fase de selección, pero con el mismo objetivo que en los otros casos: comprobar si las universidades con prestigio a nivel académico ofrecen la información sobre la universidad a través de su web de tal for- ma que pueda ser visitada por todas las personas (profesores, estudiantes), independientemente de si tiene o no algún tipo de discapacidad, es decir si son accesibles.

\section{SELECCIÓN DE UNIVERSIDADES}

Como ya se ha indicado, el objetivo principal del trabajo presentado en este artículo ha sido realizar una evaluación de la accesibilidad de páginas web de una selección de universidades españolas y extranjeras que aparecen en los rankings universitarios internacionales, con el fin de comprobar si las mejores universidades tienen páginas web accesibles. En este tipo de rankings se presenta una clasificación comparativa entre instituciones universitarias de distintos países (Rodríguez, 2006). A la hora de realizar estos rankings se suele diferenciar entre dos grandes familias: (1) Las listas "reputacionales" o subjetivas, elaboradas con base en encuestas de opinión sobre grupos que son capaces de emitir puntos de vista relevantes (Federkeil, 2008). Este método es el más antiguo y suele ser criticado por problemas de representatividad estadística. (2) Los Ilamados "rankings objetivos", basados en indicadores de desempeño que se calculan a partir de un determinado conjunto de datos empíricos (Dill y Soo, 2005). Las clasificaciones del segundo tipo son los que más abundan, debido al hecho de que en Internet circula un gran número de datos concernientes a las variables de desempeño universitario.

Tabla III. Principales estándares y recomendaciones sobre accesibilidad de contenidos web

\begin{tabular}{lc}
\hline \multicolumn{1}{c}{ Estándar o recomendación } & Referencia \\
\hline $\begin{array}{l}\text { UNE 139803: Aplicaciones informáticas para personas con discapacidad. Requisitos de accesibilidad para } \\
\text { contenidos en la web. (Versión 2004 equivalente a WCAG 1.0, versión 2012 equivalente a WCAG 2.0) }\end{array}$ & $\begin{array}{c}\text { (AENOR, 2004) } \\
\text { (AENOR, 2012) }\end{array}$ \\
\hline UNE 139802: Requisitos de accesibilidad de software. (Traducción de ISO 9241-171) & (AENOR, 2009) \\
\hline BS 8878: Web accessibility. Code of practice & (BSI, 2010) \\
\hline $\begin{array}{l}\text { CWA 15554: Specifications for a Web Accessibility Conformity Assessment Scheme and a Web Accessibility } \\
\text { Quality Mark }\end{array}$ & (CEN, 2006) \\
\hline $\begin{array}{l}\text { Section 508 Standards: Rules 1194.22 Web-based intranet and internet information and applications. } \\
\text { Incluye algunos requisitos WCAG 1.0) }\end{array}$ & (GSA, 2010) \\
\hline $\begin{array}{l}\text { ISO 9241-151 Ergonomics of human-system interaction -- Part 151: Guidance on World Wide Web user } \\
\text { interfaces }\end{array}$ & (ISO, 2008a) \\
\hline ISO 9241-171 Ergonomics of human-system interaction -- Part 171: Guidance on software accessibility & (ISO, 2008b) \\
\hline ISO/IEC TR 29138: Information technology - Accessibility considerations for people with disabilities & (ISO, 2009) \\
\hline $\begin{array}{l}\text { ISO 9241-210 Ergonomics of human-system interaction -- Part 210: Human-centred design for interactive } \\
\text { systems }\end{array}$ & (ISO, 2010) \\
\hline WCAG 1.0: Web Content Accessibility Guidelines & (W3C, 1999) \\
\hline WCAG 2.0: Web Content Accessibility Guidelines & (W3C, 2008) \\
\hline WAI-ARIA 1.0: Accessible Rich Internet Applications & (W3C, 2011) \\
\hline WCAG-EM 1.0 Website Accessibility Conformance Evaluation Methodology & (W3C, 2012) \\
\hline
\end{tabular}


Las principales críticas a estos rankings suelen referirse a que tienden a comparar realidades institucionales muy distintas $y$, sobre todo, porque, excepto algunos indicadores básicos (tamaño de las poblaciones estudiantiles y académicas, recursos financieros de las instituciones, o indicadores de productividad científica), las variables de la calidad docente, o la apreciación de las funciones de difusión y extensión, suelen pasar desapercibidas (Federkeil, 2008; Ishikawa, 2009). Los rankings mundiales que se han utilizado en este estudio son algunos de los más conocidos y analizados en publicaciones internacionales (Aguillo y otros, 2008; Dill y Soo, 2005; Federkeil, 2008; Ishikawa, 2009; Liu y Cheng, 2005; Ordorika y Rodríguez-Gómez, 2010). Se trata de los siguientes:

- Academic Ranking of World Universities (ARWU) de la Universidad Jiao Tong de Shanghai (http://www.arwu.org). Este ranking concibe la educación superior como equivalente a la investigación científica, valorando, entre otros factores, la obtención de premios Nobel por los profesores e investigadores (Liu y Cheng, 2005). Se ha utilizado la edición de 2010.

- Times Higher Education Supplement (THES) de TSL Education del Reino Unido (http:// www.timeshighereducation.co.uk). Considera que la educación superior está asociada con la construcción de la reputación como un fin en sí misma y con referencia al mercado internacional (Ordorika y Rodríguez-Gómez, 2010). Se ha utilizado la edición 2011-2012.

- Webometrics Rankings of World Universities del Laboratorio de Cibermetría del CSIC de España (http://www.webometrics.info). Considera la productividad y el efecto de los productos académicos universitarios colocados en Internet (Aguillo y otros, 2008). Se ha utilizado la edición de enero de 2012.

Se ha decidido analizar sólo universidades que aparezcan simultáneamente en los tres rankings. En el caso de las universidades extranjeras, otro requisito que se ha considerado es que se encuentren entre las 10 primeras de al menos dos de los rankings. En la tabla IV se muestran las 9 universidades que cumplen este requisito, así como la posición ocupada en cada uno de los rankings.

En el caso de las universidades españolas, se han seleccionado las 7 únicas universidades incluidas simultáneamente en los tres rankings (Tabla V).

Tabla IV. Universidades extranjeras seleccionadas (orden alfabético)

\begin{tabular}{lccc}
\hline \multicolumn{1}{c}{ Universidad } & ARWU & THES & Webometrics \\
\hline California Institute of Techonology (Estados Unidos) & 6 & 1 & 37 \\
\hline Harvard University (Estados Unidos) & 1 & 2 & 1 \\
\hline Massachusetts Institute of Technology (Estados Unidos) & 4 & 7 & 2 \\
\hline Princeton University (Estados Unidos) & 7 & 5 & 45 \\
\hline Stanford University (Estados Unidos) & 3 & 3 & 3 \\
\hline University of California, Berkeley (Estados Unidos) & 2 & 10 & 5 \\
\hline University of Cambridge (Reino Unido) & 5 & 6 & 24 \\
\hline University of Chicago (Estados Unidos) & 9 & 9 & 60 \\
\hline University of Oxford (Reino Unido) & 10 & 4 & 36 \\
\hline
\end{tabular}

Tabla V. Universidades españolas seleccionadas (orden alfabético)

\begin{tabular}{|c|c|c|c|}
\hline Universidad & ARWU & THES & Webometrics \\
\hline Universidad Autónoma de Barcelona & $301-400$ & $201-225$ & 221 \\
\hline Universidad Autónoma de Madrid & $201-300$ & $276-300$ & 469 \\
\hline Universidad de Barcelona & $201-300$ & $201-225$ & 252 \\
\hline Universidad de Valencia & $201-300$ & $301-350$ & 268 \\
\hline Universidad de Zaragoza & $401-500$ & $351-400$ & 253 \\
\hline Universidad Politécnica de Valencia & $301-400$ & $351-400$ & 261 \\
\hline Universidad Pompeu Fabra & $301-400$ & 186 & 328 \\
\hline
\end{tabular}




\section{INDICADORES DE ACCESIBILIDAD WEB EVALUADOS}

Como en los estudios citados en la tabla II, un paso previo a la evaluación de la accesibilidad es la selección de las páginas de cada universidad, cuya accesibilidad se va a analizar. El número de páginas utilizado por cada autor es diferente; por ejemplo, Ribera y otros (2009) utilizaron 2 páginas por universidad; Alexander y Rippon (2007) analizaron 4 de cada universidad, y en el informe de Discapnet (2010) fueron 5 las evaluadas. En nuestro estudio se han seleccionado 3 páginas suficientemente representativas de cada una de las 16 universidades, según el siguiente criterio:

1. La página principal del sitio web (home) de la universidad. Es la página más importante en cuanto a accesibilidad, porque si no lo es, los usuarios podrían tener problemas incluso para llegar a otras páginas del sitio.

2. Una página que contenga formularios y que sea habitual su visita por los usuarios para hacer gestiones, dado que entre los requisitos de accesibilidad web a evaluar, hay algunos asociados a los formularios en los que los usuarios deben introducir datos o interactuar con el sistema, porque si no se ha diseñado adecuadamente puede presentar problemas de accesibilidad.

3. Una página que contenga tablas. Por la misma razón que el caso anterior, porque existen requisitos específicos de accesibilidad para este componente de las páginas web, cuyo uso es muy habitual para presentar información.

De este modo, al analizar tres páginas de cada una de las universidades se pueden obtener unos resultados más equilibrados que si únicamente analizásemos la página principal, y existe una gran probabilidad de que entre las tres incluyan la mayoría de componentes problemáticos frente a la accesibilidad. Es evidente que un estudio exhaustivo de todas las páginas de la universidad conduciría a unos resultados más precisos, pero también es un realidad que la mayoría de las páginas de un sitio web siguen un patrón de diseño similar.

Una vez seleccionada la muestra de páginas a analizar, el siguiente paso es la definición de la métrica que se evaluará para determinar el grado de accesibilidad del conjunto de páginas de cada universidad. Normalmente dicha métrica se calcula a partir de indicadores derivados de los requisitos establecidos en estándares, como es el caso de WCAG. Por ejemplo, en los informes de Discapnet, incluido el de universidades de España (Discapnet, 2010) se han utilizado 12 indicadores, que coincidían con los criterios de éxito de WCAG 1.0. Por su parte, Ribera y otros, (2009), además de los indicadores WCAG 1.0 utilizaron los añadidos en la legislación de Estados Unidos (GSA, 2010) y otros 5 nuevos definidos por los autores del estudio. En el estudio de Hill (2010) se utilizaron 7 indicadores específicos para la información en la web de las universidades, pero sin considerar WCAG en su definición. En el análisis de la accesibilidad web de universidades norteamericanas realizadas por Thomson y otros. (2010) se definieron 8 indicadores. En general se trata de indicadores derivados de WCAG 1.0. En nuestro caso, como han hecho otros autores (Hernández y Amado-Salvatierra, 2012,; Karhu y otros, 2012), para realizar la evaluación de cada una de las páginas de la muestra, se ha tomado como marco de referencia las Pautas WCAG 2.0, sintetizadas en una serie de verificaciones técnicas de aquellos aspectos más relevantes y con mayor incidencia. Basándose en el estudio realizado por el INTECO (2008), estas verificaciones se reúnen en un conjunto de catorce indicadores referidos a las recomendaciones WCAG.

Estos indicadores han sido seleccionados debido a que reflejan la mayoría de las WCAG $2.0 \mathrm{co-}$ rrespondientes a los tres niveles posibles ( $A, A A$ y AAA). Los indicadores están formados a su vez por una serie de comprobaciones que analizan diferentes aspectos del indicador. Se considera que estos criterios son capaces de proporcionar una idea general bastante ajustada de la accesibilidad de un sitio web.

Los indicadores que se han tenido en cuenta para realizar el análisis, son los que se describen a continuación:

1. Documentos web válidos: La validación de los documentos frente a las gramáticas formales declaradas en los mismos permite asegurar la compatibilidad entre los distintos agentes de usuario utilizados para acceder al contenido, al tiempo que facilitan una presentación uniforme.

- Documentos HTML válidos: Se comprueba que todos los documentos son válidos respecto a la gramática declarada, utilizando, en su defecto, una gramática "HTML 4.01 Transitional".

- Documentos CSS válidos: Se comprueba que la información de estilos (presentación, maquetación y posicionamiento) es válida frente a la gramática declarada en la especificación de CSS 2.1 .

2. Imágenes: Las imágenes deben disponer de una descripción textual alternativa que asegure la comprensión del documento cuando éstas no se muestran, o cuando no se pueden visualizar. Se tienen en cuenta las siguientes alternativas:

- Imágenes con texto alternativo: Existencia de un texto alternativo para las imágenes.

- Ausencia de imágenes con texto: No se deben utilizar imágenes para transmitir 
información textual cuando ésta es representable mediante (X)HTML y CSS. Los logotipos de la página se consideran una excepción.

- Mapas de imagen con texto alternativo: Existencia de un texto alternativo para las diferentes áreas activas de los mapas de imagen.

3. Encabezados: Debe existir una estructura de encabezados que refleje adecuadamente la estructura lógica de los documentos, de forma que faciliten la lectura, comprensión y navegación no visual de los mismos.

- Encabezado de primer nivel: Existencia de un encabezado de nivel superior ("H1") como encabezado principal del documento.

- Existencia de encabezados: Existencia de encabezados a lo largo de todo el documento, con un correcto anidamiento, asegurando que se mantiene la correcta jerarquía en el documento.

4. Enlaces: Como elemento fundamental en la estructura e interacción con un sitio web, la correcta utilización de los enlaces resulta fundamental para garantizar el uso completo y satisfactorio de un sitio web, así como para conocer el objetivo y propósito de los mismos, teniendo en cuenta:

- Ausencia de enlaces sin contenido.

- Ausencia de enlaces con el mismo texto y destinos diferentes.

- Ausencia de enlaces que se abran en ventanas nuevas: Se debe evitar la apertura de nuevas ventanas ya que el usuario puede perder el punto de referencia en la navegación y sentirse confuso. No obstante, en caso de realizarse se debe informar convenientemente al usuario mediante el texto del enlace o un icono representativo.

\section{Contraste y uso semántico de los colores:}

- Contraste mínimo: Debe existir un contraste suficiente entre el color de primer plano y el color de fondo, en especial en las imágenes que transmiten información textual. La presentación visual del texto y las imágenes de texto deben tener una relación de contraste de al menos 4.5:1, excepto para los textos que son pura decoración, logotipos o textos e imágenes de texto a gran tamaño (deberá cumplir una relación de contraste de al menos 3:1).

- Contraste mejorado: Debe existir un contraste suficiente entre el color de primer plano y el color de fondo, en especial en las imágenes que transmiten información textual. La presentación visual del texto y las imágenes de texto deben tener una relación de contraste de al menos 7.1, excepto para los textos que son pura decoración, logotipos o textos e imágenes de texto a gran tamaño (deberá cumplir una relación de contraste de al menos 4.5:1).

- Empleo del color: El color no se debe emplear como el único medio visual para transmitir información, indicar una acción o distinguir visualmente un elemento.

- Características sensoriales: Las instrucciones que han de proporcionarse para comprender y operar con un contenido no deben basarse solamente en las características sensoriales, tales como forma, tamaño, ubicación visual, orientación o sonido.

6. Presentación: La separación de los aspectos referentes a presentación y contenido ha de permitir la comprensión del documento incluso cuando se prescinde totalmente de la información visual. En este aspecto se tienen en cuenta las siguientes comprobaciones:

- Ausencia de marcado presentacional: Se comprueba que no se usen elementos o atributos de presentación en el código HTML.

- Ausencia de tablas para realizar la maquetación: El uso de las tablas de datos con esta finalidad dificulta en gran medida la comprensión del sitio para los usuarios que naveguen, por ejemplo, a través de un lector de pantalla. En su lugar se deben usar hojas de estilo CSS

Presentación visual: Para los bloques de texto del documento se deben cumplir las cinco características siguientes:

- El usuario puede cambiar los colores de primer plano y de fondo de la página por los que él elija.

- El ancho de línea no pueda exceder los 80 caracteres.

- El texto no puede estar justificado.

- El interlineado (espacio entre líneas) tiene que ser al menos un espacio y medio en el interior de los párrafos.

- El texto puede ampliarse hasta un 200 por ciento, de manera que el usuario no necesite desplazarse horizontalmente para leer una línea de texto en una ventana a pantalla completa. 
7. Tamaño del texto: El texto debe estar definido en unidades relativas de forma que se permita su redimensionado para facilitar su legibilidad, adaptándose a las necesidades de las personas que acceden a él.

- Uso de unidades relativas: Se deben usar unidades relativas para el tamaño del texto, tanto, en las hojas de estilo vinculadas como en los estilos "en línea".

8. Formularios: Los elementos de formulario para la introducción de datos han de usarse correctamente, de forma que permitan una interacción adecuada con las ayudas técnicas y usuarios.

- Método estándar de envío: Se verifica que el mecanismo de envío de los formularios se base en la existencia de un botón de tipo submit y no mediante métodos alternativos no accesibles.

- Existencia de etiquetas: Existencia de asociación entre las etiquetas y los controles del formulario. También se comprueba si los controles de selección están agrupados.

9. Tablas de datos: Estos elementos han de utilizarse de forma correcta para identificar datos tabulares y relacionados. No deben utilizarse para organizar la presentación y maquetación de los contenidos.

- Presencia de título: Los resúmenes que describen el propósito de una tabla o el título de la misma, pueden ser localizados por un motor de búsqueda.

- Asociación entre los encabezados y las celdas de la tabla.

- Uso de elementos para tablas de datos en tablas de maquetación.

10. Accesibilidad a través de teclado: Los componentes de la interfaz de usuario y la navegación deben ser operables, por lo que es necesario hacer que toda la funcionalidad de la página esté disponible a través del teclado.

- Funcionalidad del teclado: Toda funcionalidad del contenido es operable a través del teclado sin que exista un límite de tiempo específico para realizar las pulsaciones de las teclas.

- Sin trampas: Es necesario poder moverse por todos los componentes de la página a través del teclado y también moverse fuera de dicho componente empleando la misma interfaz de teclado.

11. Destellos: La intención de este criterio de cumplimiento es permitir a los usuarios un acceso completo al sitio sin inducirle convulsiones a causa de la fotosensibilidad. Los usua- rios que sufren de desórdenes convulsivos por fotosensibilidad pueden sufrir ataques debido al contenido que destella varias veces con cierta frecuencia. Algunas personas son aún más sensibles a los destellos rojos que a otros colores, por lo tanto se proporciona una prueba especial para los destellos con rojo saturado.

- Tres destellos o por debajo del umbral: Las páginas web no deben contener nada que destelle más de tres veces en un segundo, o el destello debe estar por debajo de los umbrales de destello general y de destello rojo.

- Tres destellos: Las páginas web no han de contener elementos que destellen más de tres veces en un segundo.

12. Navegable: Es necesario crear páginas web que proporcionen medios que sirvan de ayuda a los usuarios a la hora de navegar.

- Saltar bloques: Existe un mecanismo que permite saltar bloques de contenido que se repiten en múltiples páginas web.

- Página titulada: Las páginas web tienen títulos significativos que describen su tema o propósito.

- Foco visible: Cualquier interfaz de usuario que sea operable a través de teclado cuenta con un indicador visible del foco de teclado.

- Múltiples medios: Existe más de un medio para localizar una página web dentro de una colección de páginas web. Se ha de comprobar que la página web tiene un link a la página principal.

- Ubicación: Se proporciona al usuario información de orientación sobre su ubicación dentro de una colección de páginas web, por ejemplo, indicando la ruta que ha seguido hasta llegar a la página indicada o marcando la posición en los menús.

13. Comprensible: Identificar correctamente el idioma y los cambios de idioma en una página facilita la comprensión del contenido a los usuarios que utilizan lectores de pantalla o programas de síntesis de voz, ya que éstos detectarán el idioma y verbalizarán correctamente el contenido.

- Idioma de la página: Se ha de identificar el idioma principal del documento mediante los atributos lang (HTML) y/o $\mathrm{xml}$ :lang (gramáticas basadas en $\mathrm{xml}$ ) en la cabecera de la página web.

- Cambios en el idioma: Los cambios de idioma deber ser marcados a través de los atributos lang y/o xml:lang de HTML y XHTML respectivamente. Excepcionalmente no es necesario marcar el cambio 
de idioma en nombres propios y palabras empleadas comúnmente en su idioma original.

14. Tiempo suficiente: Debe darse a los usuarios el tiempo suficiente para leer y usar un contenido. Estos criterios ayudan a que los usuarios pueden completar una tarea sin cambios inesperados en el contenido, que sean resultados de un límite de tiempo o de una información cambiante.

- Límite de tiempo ajustable: Permitir que, por cada límite de tiempo establecido en la página, el usuario pueda desactivarlo o variarlo.

- Pausar, detener, ocultar: Para cualquier información que se mueva, parpadee, se desplace o se actualice automáticamente, se tiene que cumplir que dicho contenido pueda ser pausado u ocultado.

Para evaluar cada uno de estos puntos hemos tenido en cuenta uno o varios criterios de éxito de las WCAG 2.0. En la tabla VI se muestran cada uno de los puntos que hemos verificado, con sus criterios de éxito y prioridades correspondientes (W3C, 2008).

Basándose en el estudio realizado por INTECO (2008), las comprobaciones se evalúan en base a los valores "Bien", "Mal", "Pocos fallos" y "No aplicable (NA)":

- Bien: Se cumplen los requisitos establecidos para la comprobación.

- Mal: No se cumplen los requisitos establecidos para la comprobación.
- Pocos fallos: Situación excepcional aplicable a comprobaciones en las que el incumplimiento es mínimo. Esta situación se valora como medio punto.

- No aplicable: No existen elementos para realizar la comprobación.

El número total de indicadores que se han evaluado es:

Total de $N^{\circ}$ indicadores $=N^{\circ}$ indicadores evaluados $x$ No páginas evaluadas

Siendo el Número de indicadores evaluados igual a 14 (los indicadores descritos en este mismo apartado) y el Número de páginas evaluadas igual a 3. Por lo tanto, el total de indicadores tenidos en cuenta en la evaluación de cada universidad es de 42.

De este número es necesario eliminar los indicadores no aplicables (NA). Para cada una de las páginas este número tomará un valor distinto. Una vez que hemos hallado el dato anterior, el porcentaje de éxito de la página se calculará de la siguiente forma:

Porcentaje de éxito $=100 \% \times$ Bien $+50 \% \times$ Pocos fallos / Total indicadores aplicables

Siendo Bien los indicadores que cumplen los requisitos establecidos por los criterios de éxito de WCAG 2.0, Pocos fallos los de incumplimiento mínimo, y Total_indicadores_aplicables el valor calculado anteriormente (42 - NA). En el caso de que se cumpliesen el total de indicadores, entonces el porcentaje de éxito de la página sería del $100 \%$.

Tabla VI. Criterios de éxito WCAG 2.0 a verificar para cada indicador de accesibilidad

\begin{tabular}{lccc}
\hline \multirow{2}{*}{ Indicador de accesibilidad a verificar } & \multicolumn{2}{c}{ Criterios de éxito WCAG 2.0 } \\
\cline { 2 - 4 } 1. Validación de las tecnologías W3C & Prioridad 1 & Prioridad 2 & Prioridad 3 \\
\hline 2. Imágenes & 4.1 .1 & & 1.4 .5 \\
\hline 3. Encabezados & 1.1 .1 & 2.4 .10 \\
\hline 4. Enlaces & $1.3 .1,2.4 .1$ & $2.4 .9,3.2 .5$ \\
\hline 5. Contraste y uso semántico de los colores & 2.4 .4 & 1.4 .6 \\
\hline 6. Presentación visual & $1.3 .3,1.4 .1,1.4 .3$ & 1.4 .8 \\
\hline 7. Tamaño del texto & 1.3 .1 & 3.3 .5 \\
\hline 8. Formularios & & & \\
\hline 9. Tablas de datos & $1.1 .1,1.3 .1,3.3 .1,3.3 .2$ & 3.3 .3 & 2.1 .3 \\
\hline 10. Accesible a través de teclado & 1.3 .1 & & 2.3 .2 \\
\hline 11. Destellos & $2.1 .1,2.1 .2$ & 2.4 .8 \\
\hline 12. Navegable & 2.3 .1 & & \\
\hline 13. Comprensible & $2.4 .1,2.4 .2$ & $2.4 .5,2.4 .7$ \\
\hline 14. Tiempo suficiente & 3.1 .1 & 3.1 .2 & \\
\hline
\end{tabular}




\section{HERRAMIENTAS UTILIZADAS PARA LA EVALUACIÓN}

En la actualidad existen herramientas disponibles para la evaluación y comprobación de la accesibilidad de una página web, que permiten automatizar el proceso de evaluación de la accesibilidad de la página, valorando su nivel de conformidad de acuerdo a las pautas WCAG 2.0. Sin embargo, la evaluación de la accesibilidad web requiere también la revisión mediante pruebas manuales, ya que las validaciones automáticas no permiten comprobar el total cumplimiento de los sitios web. Sólo así es posible verificar el cumplimiento de ciertos indicadores como la adecuación de los textos alternativos en las imágenes o la comprensibilidad del texto de los enlaces, entre otros. Por este motivo, el proceso de evaluación de la accesibilidad web seguido ha constado de dos fases:

1. En primer lugar, se ha realizado un análisis automático para detectar algunos problemas de accesibilidad. Las herramientas utilizadas no emplean ningún método de inteligencia artificial y sus resultados se obtienen de forma mecánica, por lo que son simplemente una ayuda y no son suficientes para asegurar que un sitio web es $100 \%$ accesible. Se han utilizado las herramientas de análisis automático indicadas en la tabla VII, disponibles en las direcciones URL indicadas.

2. Como complemento, se ha realizado una evaluación manual para identificar todos aquellos problemas que no pueden ser comprobados en la primera fase, y se han revisado aquellos puntos dudosos que requieren de pruebas adicionales para su comprobación completa. Esta evaluación también se basa en la utilización de herramientas que simulan el entorno real en el que se va a realizar la utilización accesible de los contenidos de las páginas web.

\section{RESULTADOS OBTENIDOS}

Como se indicó en el apartado 3, se ha realizado la evaluación de la accesibilidad de 16 sitios web pertenecientes a universidades de todo el mundo incluidas simultáneamente en los rankings ARWU, THES y Webometrics. Se han seleccionado 9 universidades extranjeras y 7 españolas. En este apartado se describen las principales barreras de accesibilidad detectadas en el análisis de los portales web, en relación a las pautas WCAG 2.0.

\subsection{Resultados por sitio web}

La tabla VIII sintetiza los resultados porcentuales obtenidos en el análisis de accesibilidad para la muestra de 16 portales universitarios, en cuanto a indicadores cumplidos (columna "Bien"), no cumplidos (columna "Mal"), indicadores con pocos fallos, no aplicados (NA) y porcentaje de éxito, ordenados de mayor a menor. Cada uno de estos posibles valores se explicó en el apartado 4 . Como se puede observar, la más destacada en el cumplimiento de criterios de accesibilidad es el portal de la Universidad de Cambridge $(55,41 \%)$, y la que obtiene un menor porcentaje de éxito es la Universidad Politécnica de Valencia $(16,67 \%)$. En la tabla VIII aparecen sombreadas las universidades españolas para que se distingan de las extranjeras.

Considerado diferentes márgenes en el porcentaje de éxito, según el grado de cumplimiento de los criterios de accesibilidad, podríamos establecer, de forma similar a como hacen otros autores (Discapnet, 2010b), los siguientes niveles o categorías:

a) Nivel de accesibilidad ALTO: Sitios web con un nivel de éxito de los requisitos de accesibilidad analizados entre $100 \%$ y 70\%. Ninguna de las universidades analizadas se ha acercado a este rango de porcentajes.

Tabla VII. Herramientas de análisis automático utilizadas

\begin{tabular}{lll}
\hline \multicolumn{1}{c}{ Herramienta } & \multicolumn{1}{c}{ Tipo } & URL \\
\hline Validador (X) HTML de W3C & Validación de gramática & http://validator.w3.org/ \\
\hline Validador de CSS de W3C & Validación de gramática & http://jigsaw.w3.org/css-validator/ \\
\hline TAW & Evaluación de accesibilidad web & http://www.tawdis.net/ \\
\hline Web Developer Toolbar & Evaluación de accesibilidad web & $\begin{array}{l}\text { https://addons.mozilla.org/en-US/firefox/addon/web- } \\
\text { developer/ }\end{array}$ \\
\hline Web Accessibility Toolbar & Evaluación de accesibilidad web & http://www.visionaustralia.org.au/info.aspx?page=614 \\
\hline WCAG Contrast Checker & Evaluación de color y contraste & $\begin{array}{l}\text { https://addons.mozilla.org/en-US/firefox/addon/wcag- } \\
\text { contrast-checker/ }\end{array}$ \\
\hline Colour Contrast Analyser & Evaluación de color y contraste & http://www.visionaustralia.org.au/info.aspx?page=628 \\
\hline PEAT & Detección de epilepsia & http://trace.wisc.edu/peat/ \\
\hline Flesh & Evaluación de legibilidad & http://flesh.sourceforge.net \\
\hline Inflesz & Evaluación de legibilidad & http://www.legibilidad.com \\
\hline
\end{tabular}


b) Nivel de accesibilidad MODERADO: Sitios web con un nivel de éxito de los requisitos de accesibilidad analizados entre $\mathbf{7 0} \%$ y $\mathbf{5 0} \%$. El nivel de cumplimiento no es tan notable como en el caso anterior, aunque se considera moderado. En esta categoría se encontrarían sólo dos de las universidades extranjeras (Cambridge y Oxford) y ninguna de las españolas.

c) Nivel de accesibilidad DEFICIENTE: Sitios web con un nivel de éxito de los requisitos de accesibilidad analizados entre $\mathbf{5 0} \%$ y $\mathbf{2 5} \%$. Estos sitios web se caracterizan por tener un nivel de accesibilidad bajo, con bastantes dificultades y barreras de acceso a los contenidos para las personas con diversidad funcional. Como se puede ver en la tabla VIII, la mayoría de los sitios web analizados se encuentran en este rango de porcentajes, viéndose el bajo cumplimiento de los criterios analizados.

d) Nivel de accesibilidad MUY DEFICIENTE: Sitios web con un nivel de éxito de los requisitos de accesibilidad analizados inferior al $\mathbf{2 5 \%}$. La única universidad incluida en esta categoría es la Universidad Politécnica de Valencia, que ha obtenido un porcentaje de éxito del $16,67 \%$.

Los datos expuestos son los correspondientes a la muestra de 3 páginas seleccionadas en cada universidad. Puede ser interesante analizar también los datos de accesibilidad únicamente de la página principal de cada institución, ya que es la primera que visita un usuario, y si presenta serios problemas de accesibilidad puede condicionar la navegación por el resto de páginas del sitio web de la universidad. Por esta razón, en la tabla IX se muestran los resultados de la evaluación considerando sólo la página principal. En este caso las universidades con mejor y peor porcentaje de éxito siguen siendo las mismas que en el caso anterior, si bien la universidad de Cambridge aumenta considerablemente su porcentaje, alcanzando el nivel de alta accesibilidad para su página principal.

\subsection{Resultados por indicador de accesibilidad}

En la tabla $X$ se muestran los resultados que se obtuvieron a partir de cada uno de los indicadores analizados. Se puede ver cuántas veces son aplicados en las páginas cada uno de los indicadores (columna "Aplica") y en cuántos casos han sido aplicados bien, mal o con pocos fallos.

Como se puede ver en la tabla $\mathrm{X}$, el indicador que consigue el mayor grado de cumplimiento es el referente a los destellos, aunque éste únicamente está presente en 6 de las muestras analizadas. Siendo el más bajo el contraste y uso semántico, requisito que no es satisfecho por ninguna de las páginas analizadas.

Tabla VIII. Resultados del análisis realizado a cada uno de los portales evaluando 3 páginas webs

\begin{tabular}{|c|c|c|c|c|c|}
\hline Portal & Bien & Mal & Pocos fallos & NA & $\begin{array}{l}\text { Porcentaje de } \\
\text { éxito }\end{array}$ \\
\hline University of Cambridge (Reino Unido) & 20 & 16 & 1 & 5 & $55,41 \%$ \\
\hline University of Oxford (Reino Unido) & 18 & 17 & 2 & 5 & $51,35 \%$ \\
\hline University of Chicago (Estados Unidos) & 16 & 19 & 2 & 5 & $45,95 \%$ \\
\hline Harvard University (Estados Unidos) & 15 & 19 & 2 & 6 & $44,44 \%$ \\
\hline Universidad de Barcelona (España) & 16 & 22 & 1 & 3 & $42,31 \%$ \\
\hline $\begin{array}{l}\text { Massachusetts Institute of Technology (Estados } \\
\text { Unidos) }\end{array}$ & 15 & 21 & 1 & 5 & $41,89 \%$ \\
\hline Princeton University (Estados Unidos) & 14 & 20 & 3 & 5 & $41,89 \%$ \\
\hline Stanford University (Estados Unidos) & 14 & 22 & 1 & 5 & $39,19 \%$ \\
\hline California Institute of Techonology (Estados Unidos) & 13 & 21 & 2 & 6 & $38,89 \%$ \\
\hline University of California, Berkeley (Estados Unidos) & 12 & 23 & 1 & 6 & $34,72 \%$ \\
\hline Universidad de Autónoma de Barcelona (España) & 12 & 26 & 2 & 2 & $32,5 \%$ \\
\hline Universidad Autónoma de Madrid (España) & 12 & 25 & 0 & 5 & $32,43 \%$ \\
\hline Universidad de Zaragoza (España) & 11 & 24 & 0 & 7 & $31,43 \%$ \\
\hline Universidad Pompeu Fabra (España) & 10 & 26 & 2 & 4 & $28,95 \%$ \\
\hline Universidad de Valencia (España) & 9 & 24 & 2 & 7 & $28,57 \%$ \\
\hline Universidad Politécnica de Valencia & 6 & 30 & 0 & 6 & $16,67 \%$ \\
\hline
\end{tabular}




\section{ANÁLISIS DE LOS RESULTADOS}

A partir del análisis de los datos que se han expuesto en el apartado anterior, se pueden extraer algunas conclusiones de carácter general, como las siguientes:

- La media de los porcentajes de éxito en los criterios de accesibilidad en todas las muestras es de $37,91 \%$, lo cual no es un resultado alentador. En el caso de las universidades españolas este porcentaje de éxito se reduce al 30,41\%, y en las universidades del mundo se incrementa llegando al $43,75 \%$.

- De las 16 páginas analizadas, únicamente las dos universidades del Reino Unido consiguen superar el $50 \%$ del porcentaje de éxito. En el caso de las universidades españolas, el porcentaje de cumplimiento más alto corresponde al portal de la Universidad de Barcelona, con un $42,31 \%$, y el más bajo a la Universidad Politécnica de Valencia con un $16,67 \%$. En cuanto a las universidades de los Estados Unidos, la mejor situada es la de Chicago $(45,95 \%)$ y la peor la de Berkeley (34,72\%).

Si tenemos en cuenta sólo la página principal de cada universidad, podemos comprobar en la tabla IX que la mitad de las universidades mejora o mantiene su accesibilidad, mientras que la otra mitad lo reduce considerablemente. Esto es especialmente importante, ya que se se trata de la puerta de entrada del usuario al sitio web de la universidad.

En cuanto al porcentaje de cumplimiento general de cada uno de los indicadores de accesibilidad, por el conjunto global de las 16 universidades, los datos fundamentales que se puedan destacar son los siguientes:

- De los 14 indicadores analizados en este estudio, únicamente cuatro de ellos consiguen superar el $50 \%$ de cumplimiento.

- El indicador que consigue el mayor grado de cumplimiento es el referente a los destellos, aunque éste únicamente está presente en 6 de las muestras. Por este motivo, se considera que el indicador de mayor grado de cumplimiento en la mayoría de las páginas web es el relacionado con el tiempo de la página, con un porcentaje cercano al $96 \%$.

- En cuanto a los indicadores con un menor grado de cumplimiento destacan algunos de ellos que obtienen unos porcentajes de cumplimiento muy bajos, llegando a ser nulo en el caso del contraste y uso semántico. Ciertas deficiencias visuales, como el daltonismo, así como el progresivo deterioro que sufren los órganos visuales de cualquier persona, hacen que el indicador relacionado con los colores no deba ser considerado menor. Su no cumplimiento

Tabla IX. Resultados del análisis realizado a cada uno de los portales evaluando sólo la página web principal (home)

\begin{tabular}{|c|c|c|c|c|c|}
\hline Página principal (home) & Bien & Mal & Pocos fallos & NA & $\begin{array}{c}\text { Porcentaje de } \\
\text { éxito }\end{array}$ \\
\hline University of Cambridge (Reino Unido) & 8 & 3 & 1 & 2 & $70,83 \%$ \\
\hline Stanford University (Estados Unidos) & 7 & 5 & 0 & 2 & $58,33 \%$ \\
\hline Universidad Autónoma de Madrid (España) & 6 & 6 & 0 & 2 & $58,33 \%$ \\
\hline University of Oxford (Reino Unido) & 6 & 5 & 1 & 2 & $54,17 \%$ \\
\hline University of Chicago (Estados Unidos) & 6 & 6 & 0 & 2 & $50 \%$ \\
\hline Harvard University (Estados Unidos) & 5 & 5 & 1 & 3 & $50 \%$ \\
\hline Massachusetts Institute of Technology (Estados Unidos) & 4 & 7 & 1 & 2 & $41,67 \%$ \\
\hline Princeton University (Estados Unidos) & 4 & 7 & 1 & 2 & $41,67 \%$ \\
\hline University of California, Berkeley (Estados Unidos) & 4 & 7 & 1 & 2 & $37,50 \%$ \\
\hline Universidad de Barcelona (España) & 4 & 9 & 0 & 1 & $30,77 \%$ \\
\hline California Institute of Techonology (Estados Unidos) & 3 & 8 & 1 & 2 & $29,17 \%$ \\
\hline Universidad de Zaragoza (España) & 3 & 8 & 0 & 3 & $27,27 \%$ \\
\hline Universidad de Valencia (España) & 3 & 8 & 0 & 3 & $27,27 \%$ \\
\hline Universidad Pompeu Fabra (España) & 3 & 9 & 0 & 2 & $25 \%$ \\
\hline Universidad de Autónoma de Barcelona (España) & 2 & 10 & 1 & 1 & $19,23 \%$ \\
\hline Universidad Politécnica de Valencia & 2 & 10 & 0 & 2 & $16,67 \%$ \\
\hline
\end{tabular}


puede llevar a la pérdida de una gran parte de la información presentada en los portales, ya que muchos usuarios pueden tener dificultades, o incluso imposibilidad, de leer los textos presentes en dichas páginas. El siguiente mas bajo es el correspondiente a la presentación visual, con un $12,50 \%$.

\section{CONCLUSIONES}

La Web se creó como una red universal de conocimiento que ha supuesto un gran avance en cuanto a la adquisición de información. Partiendo de la idea de que hoy en día, debido a diferentes motivos, existen grandes barreras en la web para un amplio número de ciudadanos, se ha realizado un estudio en profundidad en las páginas de universidades importantes. A partir de los resultados obtenidos, se ha llegado a la conclusión de que la mayoría de los sitios analizados no llegan a un nivel de cumplimiento aceptable. A pesar de ser todas ellas instituciones incluidas en tres importantes rankings universitarios, únicamente dos de las universidades analizadas, lo que representa un $12,5 \%$ de la muestra, consigue un nivel de accesibilidad moderado. Estas barreras pueden dificultar o impedir el acceso a los contenidos a personas que puedan tener alguna limitación física o sensorial.

Estos resultados no difieren en gran medida con los obtenidos en otros estudios similares, como por ejemplo el publicado por Discapnet (2010b), en el que se analiza la accesibilidad web de 16 portales universitarios españoles: 14 universidades, el portal del Ministerio de Educación, y el portal de la Conferencia de Rectores (CRUE). Sólo 2 de las universidades analizadas en este artículo también es- taban incluidas en el estudio de Discapnet, la Universidad de Barcelona y Universidad de Valencia. Aunque los resultados obtenidos en ambos casos no han sido los mismos, debido a que los criterios de evaluación han sido diferentes. En el caso de la Universidad de Barcelona, según el método de Discapnet, el porcentaje de éxito de cumplimiento de criterios de accesibilidad WCAG 1.0 en 2010 era del 54,29\% (en nuestro caso, aplicando WCAG 2.0 se ha obtenido un $42,31 \%$ ). La Universidad de Valencia obtuvo un $24,40 \%$, muy parecido al $28,57 \%$ de nuestro estudio.

Otro estudio de accesibilidad web de todas las universidades españolas fue el realizado por Ribera y otros (2009). En dicho trabajo se evaluaba el porcentaje de cumplimiento de los indicadores WCAG 1.0 por la página principal de cada universidad en 2006. Aunque en nuestro estudio también se ha incluido dicha página, no es posible hacer una comparación entre ambos, por basarse en criterios diferentes. Aunque sí se puede asegurar, a la vista de las cifras de dicho trabajo, que al menos las dos universidades españolas con página principal mejor posicionadas en nuestro estudio (Universidad Autónoma de Madrid y Universidad de Barcelona), han mejorado su nivel de accesibilidad desde el año 2006.

En cuanto a otros estudios internacionales de accesibilidad web en los que hayan estado implicadas algunas de las universidades extranjeras citadas en este artículo, de la información disponible, sólo en el de Hill (2010) se incluyó la Universidad de Harvard, con un porcentaje de cumplimiento de los indicadores de accesibilidad de un $43 \%$, muy pare-

Tabla X. Resultados obtenidos del análisis clasificados por indicador verificado

\begin{tabular}{|c|c|c|c|c|c|}
\hline Indicador de accesibilidad & Aplica & Bien & Mal & $\begin{array}{l}\text { Pocos } \\
\text { fallos }\end{array}$ & $\begin{array}{c}\text { Porcentaje de } \\
\text { éxito }\end{array}$ \\
\hline Destellos & 6 & 6 & 0 & 0 & $100 \%$ \\
\hline Tiempo suficiente & 48 & 46 & 2 & 0 & $95,83 \%$ \\
\hline Accesibilidad a través de teclado & 48 & 29 & 19 & 0 & $60,42 \%$ \\
\hline Encabezados & 48 & 26 & 21 & 1 & $55,21 \%$ \\
\hline Comprensible & 48 & 22 & 26 & 0 & $45,83 \%$ \\
\hline Enlaces & 48 & 20 & 24 & 4 & $45,83 \%$ \\
\hline Imágenes & 48 & 12 & 22 & 14 & $39,58 \%$ \\
\hline Navegable & 48 & 16 & 31 & 1 & $34,37 \%$ \\
\hline Formularios & 37 & 9 & 26 & 2 & $27,03 \%$ \\
\hline Tamaño del texto & 48 & 10 & 38 & 0 & $20,83 \%$ \\
\hline Tablas & 20 & 4 & 16 & 0 & $20,00 \%$ \\
\hline Documentos válidos & 47 & 7 & 40 & 0 & $14,89 \%$ \\
\hline Presentación & 48 & 6 & 42 & 0 & $12,50 \%$ \\
\hline Contraste y uso semántico & 48 & 0 & 48 & 0 & $0 \%$ \\
\hline
\end{tabular}


cido al obtenido en nuestro estudio (44\%), a pesar de utilizar diferentes indicadores.

El trabajo presentado en este articulo tiene la novedad de incluir universidades españolas y extranjeras, por lo que merece la pena comentar brevemente alguna conclusión derivada de la comparación de los resultados obtenidos por ambos grupos. A nivel de sitio web, considerando la muestra de tres páginas relevantes por universidad, todas las webs españolas tienen una accesibilidad menor que las extranjeras, excepto en el caso de la Universidad de Barcelona, que tiene un nivel de accesibilidad por encima de 5 de las 9 universidades extranjeras analizadas, sólo por detrás de Cambridge, Oxford, Chicago y Harvard; y por delante de universidades tan importantes como Massachusetts, Princeton, Stanford, California y Berkeley.

Si tenemos en cuenta sólo la página principal de las universidades, entonces la Universidad Autónoma de Madrid sería la mejor posicionada, solo por detrás de las universidades de Cambridge y Stanford. Y la Universidad de Barcelona sólo superaría en este caso a la de California. El resto de universidades españolas estaría por detrás de las extranjeras en nivel de accesibilidad de su página web principal. Después de analizar la cifras, se puede afirmar que aunque en general las universidades españolas incluidas simultáneamente en los rankings internacionales ARWU, THES o Webometrics, tienen páginas web menos accesibles que las mejores posicionadas en los rankings, algunas de ellas tienen un nivel de accesibilidad similar o incluso superior

Para concluir, es conveniente aclarar respecto a la accesibilidad web que, aunque es habitual que se piense que la accesibilidad es algo que atañe únicamente a personas discapacitadas, se trata de una idea equivocada, ya que cualquier persona puede tener dificultades de acceso a la Web en un momento determinado, por ejemplo, debido a una conexión de Internet lenta, a dificultades para entender el idioma o a la fractura de una extremidad superior. En definitiva, conseguir sitios web accesibles, además de ser un imperativo legal en algunos países, es muy conveniente porque es algo que beneficia a todos los usuarios.

En nuestra opinión, debido a que el acceso a las tecnologías de la información es determinante en la sociedad en la que vivimos, los diseñadores de páginas web deberían plantearse crear sus sitios adaptándose a las normas de accesibilidad, como WCAG, olvidando prejuicios tales como la creencia de que crear páginas accesibles supone una mayor dificultad o inversión. Se trata de conseguir que todas las personas puedan acceder a ellas, ya que la falta de acceso a este tipo de tecnologías genera desigualdades y discriminación en nuestra sociedad. Pero como afirma Terrill Thomson (Thomson y otros, 2010), en referencia a la accesibilidad web de las universidades norteamericanas, pero que se puede aplicar también tanto a las españolas como a las de otros países, "la realidad es que todavía queda un largo camino por recorrer".

\section{AGRADECIMIENTOS}

El trabajo ha sido realizado con el apoyo de la Comisión Europea a través del proyecto ESVI-AL del programa ALFA III.

\section{BIBLIOGRAFÍA}

AENOR (2004). UNE 139803:2004. Requisitos de accesibilidad para contenidos en la web. Madrid: Asociación Española de Normalización y Certificación.

AENOR (2009). UNE 139802:2009. Requisitos de accesibilidad al ordenador: Software. Madrid: Asociación Española de Normalización y Certificación.

AENOR (2012). UNE 139803:2012. Requisitos de accesibilidad para contenidos en la web. Madrid: Asociación Española de Normalización y Certificación.

Aguillo, I.F.; Ortega, J.L.; Fernández, M. (2008). Webometric Ranking of World Universities: Introduction, Methodology, and Future Developments. Higher Education in Europe, vol. 33(2-3), 233-244.

Alexander, D.; Rippon, S. (2007). University website accessibility revisited [en línea]. Australia: Southern Cross University. http://ausweb.scu.edu.au/aw07/ papers/refereed/alexander/paper.html [consulta: 17-05-2012].

BOE (2003). Ley 51/2003, de 2 de diciembre, de igualdad de oportunidades, no discriminación y accesibilidad universal de las personas con discapacidad (BOE número 289 de 3/12/2003, páginas 43187 a 43195).

BOE (2007a). Real Decreto 1494/2007, de 12 de noviembre (Ministerio de la Presidencia), por el que se aprueba el Reglamento sobre las condiciones básicas para el acceso de las personas con discapacidad a las tecnologías, productos y servicios relacionados con la sociedad de la información y medios de comunicación social (BOE de 21/11/2007, páginas 47567 a 47572).

BOE (2007b). Ley 49/2007, de 26 de diciembre, por la que se establece el régimen de infracciones y sanciones en materia de igualdad de oportunidades, no discriminación y accesibilidad universal de las personas con discapacidad (BOE número 310 de 27/12/2007, páginas 53278 a 53284 ).

BOE (2007c). Ley 56/2007, de 28 de diciembre, de Medidas de Impulso de la Sociedad de la Información (BOE número 312 de 29/12/2007, páginas 53701 a 53719).

Brajnik, G. (2008). A Comparative Test of Web Accessibility Evaluation Methods. Proceedings of the 10th International ACM SIGACCESS Conference on Computers and Accessibility, Nueva York: ACM, 113-120.

Brajnik, G. (2009). Validity and reliability of web accessibility guidelines. Proceedings of the 11 th international ACM SIGACCESS conference on Computers and Accessibility, Nueva York: ACM, 131-138.

BSI (2010). BS 8878: 2010 Web accessibility code of practice. Londres: British Standards Institution.

Caballero-Cortés, L.; Faba-Pérez, C; Moya-Anegón F. (2009). Evaluación comparativa de la accesibilidad 
de los espacios web de las bibliotecas universitarias españolas y norteamericanas. Investigación Bibliotecológica: archivonomía, bibliotecología e información, vol. 47(23), 45-66.

CEN (2006). CWA 15554: Specifications for a Web Accessibility Conformity Assessment Scheme and a Web Accessibility Quality Mark. European Committee for Standardization.

CERMI (2010). Observatorio de Accesibilidad de las Páginas de Internet de las principales empresas españolas. $2^{a}$ entrega 2010 [en línea]. Madrid: Comité Español de Representantes de Personas con Discapacidad . http://www.discapnet.es/Castellano/areastematicas/Accesibilidad/Observatorio_infoaccesibilidad/Otros_Informes/Documents/ CERMI-ObservatorioWebEsp-Parte2_30042010.pdf [consulta: 17-05-2012].

Comisión Europea (2008). Assessment of the Status of eAccessibility in Europe [en línea]. Bruselas: Europe's Information Society Thematic Portal. http:// ec.europa.eu/information_society/activities/einclusion/library/studies/meac_study/index_en.htm [consulta: 17-05-2012]

Comisión Europea (2009a). Accessibility of European Commission Websites - Analysis of current status and recommendations for improvement [en línea]. Bruselas: Europe's Information Society Thematic Portal. http://ec.europa.eu/information_society/ activities/einclusion/policy/accessibility/web_access/commission_web_accessibility/index_en.htm [consulta: 17-05-2012].

Comisión Europea (2009b). Study report: Web accessibility in European countries: level of compliance with latest international accessibility specifications, notably WCAG 2.0, and approaches or plans to implement those specifications [en línea]. Bruselas: Europe's Information Society Thematic Portal. http://ec.europa.eu/information_society/activities/ einclusion/library/studies/web_access_compliance/ index_en.htm [consulta: 17-05-2012].

Córdova, M.A. (2012). Estudio comparativo de accesibilidad web en portales informativos de universidades peruanas de educación a distancia. En Calidad y Accesibilidad de la Formación Virtual. Alcalá de Henares: Universidad de Alcalá, 63-73.

Dill, D.; Soo, M. (2005). Academic Quality, League Tables, and Public Policy: A crossnational analysis of University Ranking Systems. Higher Education Review, vol. 49(4), 495-533.

Discapnet (2009a). Accesibilidad web en los portales de los Ministerios [en línea]. Madrid: Discapnet. http://www.discapnet.es/Castellano/areastematicas/Accesibilidad/Observatorio_infoaccesibilidad/ informesInfoaccesibilidad/Paginas/AccesibilidaddePortalesWebUniversitarios.aspx [consulta: 1705-2012].

Discapnet (2009b). Accesibilidad en los portales web de Servicios y Plataformas de eLearning [en línea]. Madrid: Discapnet. http://www.discapnet.es/Castellano/areastematicas/Accesibilidad/Observatorio infoaccesibilidad/informesInfoaccesibilidad/Paginas/ AccesibilidadenlosportalesWebdeServiciosyPlataformasdeeLearning.aspx [consulta: 17-05-2012].
Discapnet (2010). Accesibilidad de Portales web Universitarios [en línea]. Madrid: Discapnet. http:// www.discapnet.es/Castellano/areastematicas/ Accesibilidad/Observatorio_infoaccesibilidad/informesInfoaccesibilidad/Paginas/AccesibilidaddePortalesWebUniversitarios.aspx [consulta: 1705-2012].

Discapnet (2011). Accesibilidad de Plataformas de Redes Sociales [en línea]. Madrid: Discapnet. http://www.discapnet.es/Castellano/areastematicas/Accesibilidad/Observatorio_infoaccesibilidad/ informesInfoaccesibilidad/Paginas/AccesibilidaddePlataformasdeRedesSociales.aspx [consulta: 1705-2012].

Federkeil, G. (2008). Rankings and Quality Assurance in Higher Education. Higher Education in Europe, vol. 33(2/3), 219-231.

GSA (2010). Section 508 Standards Guide [en línea]. Estados Unidos: General Services Administration. http://www.section508.gov/docs/Section $\% 20$ $508 \% 20$ Standards\%20Guide.pdf [consulta: 1705-2012].

Hernández, R.; Amado-Salvatierra, H.R. (2012). Evaluación de la accesibilidad de portales web en instituciones educativas en el área de Centroamérica. En Calidad y Accesibilidad de la Formación Virtual. Alcalá de Henares: Universidad de Alcalá, 74-80.

Hill, C.M. (2010). Access to Information for Students with Disabilities; An Evaluation of the 2010 U.S. News and World Report's Ranking of the Nation's Best Colleges and Universities. UIW McNair Scholars Research Journal, vol. 11, 100-115.

INTECO (2008). Estudio-Diagnóstico de accesibilidad y calidad web en la pyme española [en línea]. Madrid: Instituto Nacional de Tecnologías de la Comunicación. http://www.inteco.es/Accesibilidad/estudios de_accesibilidad/accesibilidad_publicaciones/ accesibilidad_estudios_e_informes/estudio_ accesibilidad_pyme [consulta: 17-05-2012].

INTECO (2009). Informe sobre la accesibilidad de los sistemas de mensajería instantánea [en línea]. Madrid: Instituto Nacional de Tecnologías de la Comunicación. http://www.inteco.es/Accesibilidad/estudios de accesibilidad/accesibilidad_publicaciones/ accesibilidad_estudios_e_informes/informe_im [consulta: 17-05-2012].

Ishikawa, M. (2009). University Rankings, Global Models, and Emerging Hegemony: Critical analysis from Japan. Journal of Studies in International Education, vol. 13(2), 159-173.

ISO (2008a). ISO 9241-151 Ergonomics of humansystem interaction -- Part 151: Guidance on World Wide Web user interfaces. Ginebra: International Organization for Standardization.

ISO (2008b). ISO 9241-171 Ergonomics of humansystem interaction -- Part 171: Guidance on software accessibility. Ginebra: International Organization for Standardization.

ISO (2009). ISO/IEC TR 29138: Information technology - Accessibility considerations for people with disabilities. Ginebra: International Organization for Standardization. 
ISO (2010). ISO 9241-210 Ergonomics of human-system interaction -- Part 210: Human-centred design for interactive systems. Ginebra: International Organization for Standardization.

ISO (2012). ISO/IEC 40500, Information technology -W3C Web Content Accessibility Guidelines (WCAG) 2.0. Ginebra: International Organization for Standardization.

Kane, S.K.; Shulman, J.A.; Ladner, R.E. (2007). A Web Accessibility Report Card for Top International University Web Sites. Proceedings of the 2007 international cross-disciplinary conference on Web accessibility. Nueva York: ACM, 148-156.

Karhu, M.; Hilera, J.R.; Fernández, L.; Rios, R. (2012). Accessibility and readability of university websites in Finland. En Calidad y Accesibilidad de la Formación Virtual. Alcalá de Henares: Universidad de Alcalá, 151-158.

Liu, N.C.; Cheng, Y. (2005). The Academic Ranking of World Universities. Higher Education in Europe, vol. 30(2), 127-136.

Ordorika, I.; Rodríguez-Gómez, R. (2010). El ranking Times en el mercado del prestigio universitario. Perfiles Educativos XXXII, vol. 129, 8-29.

Ribera, M.; Térmens, M.; Frías, A. (2009). La accesibilidad de las webs de las universidades españolas. Balance 2001-2006. Revista Española de Documentación Científica, vol. 32(3), 66-88.
Rodríguez, R. (2006). Rankings universitarios ¿un oscuro objeto de deseo? Segunda parte. Campus Milenio, no. 202, 23.

Serrano, E.; Moratilla, A.; Olmeda, I. (2010). Métrica para la evaluación de la accesibilidad en Internet: propuesta y testeo. Revista Española de Documentación Científica, vol. 33(3), 378-396.

Thomson, T.; Burgstahler, S.; Moore, E.J. (2010). Web accessibility: A longitudinal study of college and university home pages in the northwestern United States. Disability and Rehabilitation: Assistive Technology, vol. 5(2), 108-114.

W3C (1999). Web Content Accessibility Guidelines (WCAG) 1.0 [en línea]. World Wide Web Consortium. http://www.w3.org/TR/WCAG10/ [consulta: 17-05-2012].

W3C (2008). Web Content Accessibility Guidelines (WCAG) 2.0 [en línea]. World Wide Web Consortium. http://www.w3.org/TR/WCAG/ [consulta: 17-05-2012].

W3C (2011). Accessible Rich Internet Applications (WAI-ARIA) 1.0. W3C Candidate Recommendation [en línea]. World Wide Web Consortium. http:// www.w3.org/TR/wai-aria/ [consulta: 17-05-2012].

W3C (2012). Website Accessibility Conformance Evaluation Methodology 1.0. W3C Working Draft [en línea]. World Wide Web Consortium. http:// www.w3.org/TR/WCAG-EM/ [consulta: 17-052012]. 